\title{
Medizin in Zeiten des Cyberspace
}

Nichts habe die Beziehung zwischen Arzt und Patient derart verändert wie das Internet, klagen Pamela Hartzband und Jerome Groopman vom Beth Israel Deaconess Medical Center, Harvard. So habe einer ihrer Patienten, ein Mensch mit Neigung zur Hypochondrie, sich mit seinen Rückenschmerzen nicht seinem Doktor anvertraut, sondern im Internet recherchiert, bis er zu der Überzeugung gelangte, Ursache seiner Beschwerden sei ein lebensbedrohliches Aneurysma. Eine Woche lang horchte der Mann ängstlich in sich hinein, tastete sich immer wieder selbst $a b$ und suchte dann doch den Arzt auf. Als der keinerlei Befund erheben konnte, verlor unser Hypochonder das Interesse an seinen Rückenschmerzen und ward gesund - vermutlich bis zur nächsten Internetrecherche.

\section{Surfen unter ärztlicher Aufsicht}

Man dürfe seine Patienten im Cyberspace nicht allein lassen, meinen
Hartzband und Groopman. Ein Normalsterblicher könne seriöse und unseriöse Informationsangebote nicht unterscheiden. Sicher nicht ganz unrichtig. Aber wie soll man seine Patienten im Cyberspace begleiten? Jedes Mal einen notfallmäßigen Hausbesuch starten, wenn der Patient seinen PC anschaltet?

Das dürfte sich kaum verwirklichen lassen. So suchen etwa $40 \%$ der Spanier im Internet nach medizinischen Informationen, schreiben José Joaquín Mira und Kollegen von der Universität Alicante. Und dies störe die Compliance doch sehr, stellen Mira et al. in ihrer Studie fest. Jeder dritte Arzt, den sie studienhalber befragt haben, meinte, das Internet untergrabe seine Glaubwürdigkeit und belaste die Arzt-Patienten-Beziehung. Und die restlichen zwei Drittel der Ärzte sind zunehmend damit beschäftigt, Patienten die im Internet angelesenen Diagnosen wieder auszureden.

\section{Todesnachricht per Twitter}

Aber es gibt noch eine ergiebigere Quelle für Fehlinformationen. Daniel Scanfield und Kollegen von der Columbia University haben festgestellt, dass sich Fehlinformationen per Twitter rasend schnell verbreiten. Für Twitter braucht man a) nicht einmal einen PC - es funktioniert auch per Handy und b) darf immer nur eine arg begrenzte Zahl von Zeichen gesendet werden. Nehmen wir an, Individuum A nimmt die Pille B, verschluckt sich daran und wäre um ein Haar erstickt. Die genauen Umstände sind für Twitter zu umfangreich, also wird die Meldung lauten, A wäre fast an B erstickt. Der nächste Twitterer hat noch anderes mitzuteilen, deshalb spart er sich das Wörtchen „fast“. Und schon zwitschert es durch die Twittergemeinde, dass am Medikament B schon Hunderte von Twitterern elendiglich zugrunde gegangen seien. Echt wahr, die haben das selbst getwittert.

REINHOLD MANHART

\section{0\% DER JUGENDLICHEN SCHRECKEN VOR HIRNDOPING NICHT ZURÜCK Tabletten für gute Noten}

Die Bereitschaft, geistige Leistungen mithilfe von Pillen zu optimieren, ist unter deutschen Jugendlichen groß. Eine Befragung von 1035 Schülern und 512 Studenten ergab, dass über $80 \%$ ihren „grauen Zellen“ medikamentös auf die Sprünge helfen würden, wenn es entsprechende Substanzen gäbe, die langfristig keine Schäden verursachen. Bei rund $4 \%$ der Befragten spielen mögliche Nebenwirkungen als Hemmschwelle keine Rolle; sie gaben an, bereits illegale oder verschreibungspflichtige Medikamente zur Steigerung der mentalen Leistungsfähigkeit eingenommen zu haben. Berufsschüler erlagen der Versuchung deutlich häufiger als Gymnasiasten, männliche Jugendliche häufiger als weibliche und
Schüler mit schlechten Noten häufiger als Einserkandidaten.

In der Befragung ging es um Substanzen wie den Wachmacher Modafinil, das ADHS-Medikament Methylphenidat sowie Psychostimulanzien wie Amphetamine, Ecstasy oder Kokain. Studieninitiator Prof. Klaus Lieb, Direktor der Klinik für Psychiatrie und Psychotherapie der Universität Mainz, sieht die hohe Bereitschaft zum Hirndoping als Warnzeichen: „In Zukunft könnte es Substanzen mit akzeptablem Risikoprofil geben, die gleichzeitig sehr effektiv kognitive Fähigkeiten steigern. Die dürften bei Schülern und Studenten auf fruchtbaren Boden fallen.“

EO -

Pressemitteilung der Johannes GutenbergUniversität Mainz, April 2010

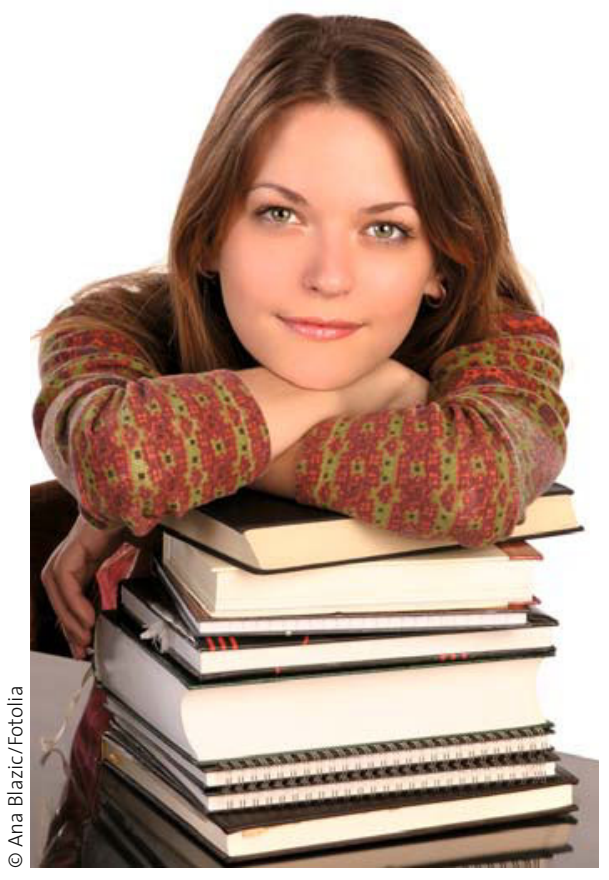

Superhirn dank Psychopharmaka? 